\title{
DISCRETE RICCATI EQUATION, HYPERGEOMETRIC FUNCTIONS AND CIRCLE PATTERNS OF SCHRAMM TYPE
}

\author{
S. I. AGAFONOV \\ Institut für Algebra und Geometrie, Martin-Luther-Universität Halle, D-06099 Halle (Saale), Germany \\ e-mail:agafonov@mathematik.uni-halle.de
}

(Received 17 December, 2003; accepted 8 July, 2004)

\begin{abstract}
Square grid circle patterns with prescribed intersection angles, mimicking holomorphic maps $z^{\gamma}$ and $\log (z)$ are studied. It is shown that the corresponding circle patterns are embedded and described by special separatrix solutions of discrete Painlevé and Riccati equations. The general solution of this Riccati equation is expressed in terms of the hypergeometric function. Global properties of these solutions, as well as of the discrete $z^{\gamma}$ and $\log (z)$, are established.
\end{abstract}

2000 Mathematics Subject Classification. 39A12, 33C99, 52C26.

1. Introduction. The theory of circle patterns is a rich and fascinating area having its origin in the classical theory of circle packings. Its fast development in recent years has been caused by the mutual influence and interplay of ideas and concepts from discrete geometry, complex analysis and the theory of integrable systems.

Progress in this area of investigation was initiated by Thurston's idea $[\mathbf{1 5}, \mathbf{2 0}]$ about approximating the Riemann mapping by circle packings. Classical circle packings comprised of disjoint open disks were later generalized to circle patterns where the disks may overlap (see for example [13]). Different underlying combinatorics were considered. Schramm introduced a class of circle patterns with the combinatorics of the square grid [19]; hexagonal circle patterns were studied in [6] and [7].

The striking analogy between circle patterns and classical analytic function theory is underlined by such facts as the uniformization theorem concerning circle packing realizations of cell complexes of a prescribed combinatorics [4], the discrete maximum principle, Schwarz's lemma [17], rigidity properties [13, 15], and the discrete Dirichlet principle [19].

The convergence of discrete conformal maps represented by circle packings was proven by Rodin and Sullivan [18]. For prescribed regular combinatorics this result was refined. He and Schramm [12] showed that for hexagonal packings the convergence is $C^{\infty}$. Uniform convergence for circle patterns with the combinatorics of the square grid and orthogonal neighboring circles was established by Schramm [19].

The approximation issue naturally leads to the question about analogues to standard holomorphic functions. Computer experiments give evidence for their existence $[\mathbf{1 1}, \mathbf{1 4}]$. However not very much is known. For circle packings with hexagonal combinatorics the only explicitly described examples are Doyle spirals $[3,10]$ which are discrete analogues of exponential maps and conformally symmetric packings, which are analogues of a quotient of Airy functions [5]. For patterns with overlapping circles more explicit examples are known: discrete versions of $\exp (z), \operatorname{erf}(z)[19], z^{\gamma}, \log (z)$ 
[2] are constructed for patterns with underlying combinatorics of the square grid; $z^{\gamma}$, $\log (z)$ are also described for hexagonal patterns $[6,7]$.

It turns out that an effective approach to the description of circle patterns with overlapping circles is given by the theory of integrable systems (see [6, 7]). For example, Schramm's circle patterns are governed by a difference equation which is the stationary Hirota equation (see $[\mathbf{1 9}, \mathbf{2 1}]$ ). This approach proved to be especially useful for the construction of discrete $z^{\gamma}$ and $\log (z)$ in $[\mathbf{2}, \mathbf{6}, \mathbf{7}]$ with the aid of an isomonodromy problem. Another connection with the theory of discrete integrable equations was revealed in [1, 2]: embedded circle patterns are described by special solutions of discrete Painlevé II equations, thus giving a geometrical interpretation thereof.

This research was motivated by the attempt to carry the results of $[\mathbf{1}, \mathbf{2}]$ over to square grid circle patterns with prescribed intersection angles giving Schramm patterns as a special case. Namely, we prove that such circle patterns mimicking $z^{\gamma}$ and $\log (z)$ are embedded. This turns out to be not straightforward and leads to assymptotic analysis of solutions to discrete Riccati equation. As the Riccati differential equation possesses the Painlevé property we are tempted to conclude that circle patterns are described by discrete equations with the Painleve property though there is no satisfactory generalization thereof to discrete equations.

We use the following definition for square grid circle patterns, which is slightly modified version of one from [19].

Definition 1. Let $G$ be a subgraph of the 1-skeleton of the cell complex with vertices $\mathbf{Z}+i \mathbf{Z}=\mathbf{Z}^{\mathbf{2}}$ and $0<\alpha<\pi$. A square grid circle pattern for $G$ with intersection angles $\alpha$ is an indexed collection of circles on the complex plane

$$
\left\{C_{z}: z \in V(G), C_{z} \in \mathbf{C}\right\}
$$

that satisfy:

1) if $z, z+i \in V(G)$ then the intersection angle of $C_{z}$ and $C_{z+i}$ is $\alpha$,

2) if $z, z+1 \in V(G)$ then the intersection angle of $C_{z}$ and $C_{z+1}$ is $\pi-\alpha$,

3) if $z, z+1+i \in V(G)$ (or $z, z-1+i \in V(G)$ ) then the disks, defined by $C_{z}$ and $C_{z+1+i}\left(C_{z}\right.$ and $C_{z-1+i}$ respectively) are tangent and disjoint,

4) if $z, z_{1}, z_{2} \in V(G),\left|z_{1}-z_{2}\right|=\sqrt{2},\left|z-z_{1}\right|=\left|z-z_{2}\right|=1$ (i.e. $C_{z_{1}}, C_{z_{2}}$ are tangent and $C_{z}$ intersects $C_{z_{1}}$ and $\left.C_{z_{2}}\right)$ and $z_{2}=z+i\left(z_{1}-z\right)$ (i.e. $z_{2}$ is one step counterclockwise from $z_{1}$ ), then the circular order of the triplet of points $C_{z} \cap C_{z_{1}}-C_{z_{2}}, C_{z_{1}} \cap C_{z_{2}}, C_{z} \cap C_{z_{2}}-C_{z_{1}}$ agrees with the orientation of $C_{z}$.

The intersection angle is the angle at the corner of the disc intersection domain (Fig. 1).

To visualize the analogy between Schramm's circle patterns and conformal maps, consider regular patterns composed of unit circles and suppose that the radii are being deformed to preserve the intersection angles of neighboring circles and the tangency of half-neighboring ones. Discrete maps taking the intersection points and the centers of the unit circles of the standard regular patterns to the respective points of the deformed patterns mimic classical holomorphic functions, the deformed radii being analogous to $\left|f^{\prime}(z)\right|$ (see Fig. 2).

In section 2 we give a definition of discrete $Z^{\gamma}$ as a solution to some integrable equation subject to a non-autonomous constraint. Its geometrical properties of immersion and embeddedness are expressed in terms of solutions for radii of corresponding circle patterns. 


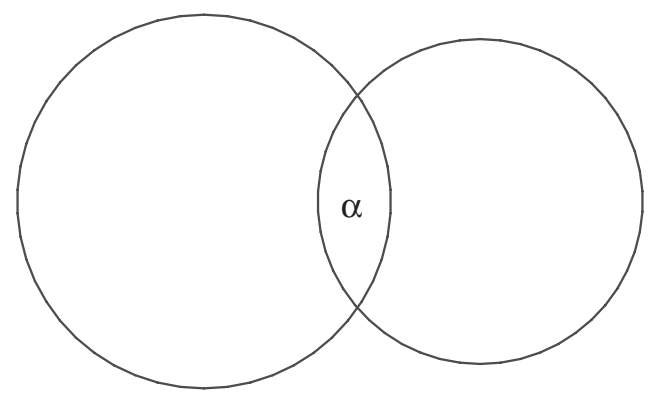

Figure 1. Circles intersection angle
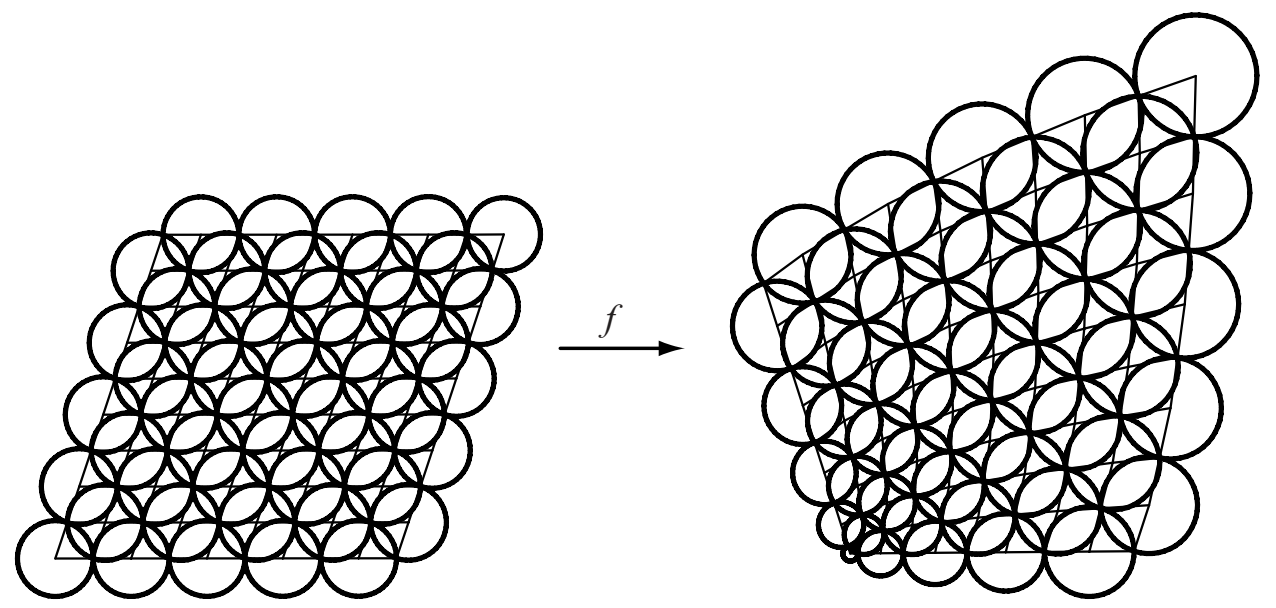

Figure 2. Schramm type circle patterns as a discrete conformal map. The discrete version of the holomorphic mapping $z^{3 / 2}$. The case $\tan \alpha=3$

For this solution to be positive it is necessary that some discrete Riccati equation has a positive solution. This equation is studied in section 3 where its general solution is expressed through the hypergeometric function.

Section 4 completes the proof of embeddednes, discrete equations of Painlevé type being the main tool. Possible generalizations for non-regular combinatorics and for non-circular patterns are discussed in section 5.

\section{Discrete $Z^{\gamma}$ and square grid circle patterns of Schramm type.}

Definition 2. The discrete map $Z^{\gamma}, 0<\gamma<2$ is the solution $f: \mathbf{Z}_{+}^{\mathbf{2}} \rightarrow \mathbf{C}$ of

$$
\begin{gathered}
q\left(f_{n, m}, f_{n+1, m}, f_{n+1, m+1}, f_{n, m+1}\right)=e^{-2 i \alpha} \\
\gamma f_{n, m}=2 n \frac{\left(f_{n+1, m}-f_{n, m}\right)\left(f_{n, m}-f_{n-1, m}\right)}{f_{n+1, m}-f_{n-1, m}}+2 m \frac{\left(f_{n, m+1}-f_{n, m}\right)\left(f_{n, m}-f_{n, m-1}\right)}{f_{n, m+1}-f_{n, m-1}},
\end{gathered}
$$

with $0<\alpha<\pi$ and the initial conditions

$$
f_{1,0}=1, \quad f_{0,1}=e^{i \gamma \alpha}
$$


where $q$ stands for the cross-ratio of elementary quadrilaterals:

$$
q\left(f_{1}, f_{2}, f_{3}, f_{4}\right)=\frac{\left(f_{1}-f_{2}\right)\left(f_{3}-f_{4}\right)}{\left(f_{2}-f_{3}\right)\left(f_{4}-f_{1}\right)} .
$$

This definition can be justified by the following properties:

- if one thinks of $f$ as defined on the vertices of the cell complex with diamondshaped faces (see Fig. 2) then (1) means that $f$ respects the cross-ratios of the faces and therefore is "locally conformal",

- the asymptotics of (2) as $n, m \rightarrow \infty$ suggest that $f$ approximates $z^{\gamma}$.

REMARK. Equation (1) with $\alpha=\pi / 2$ was used in [8] to define discrete conformal maps. The motivation was that $f$ maps vertices of squares into vertices of "conformal squares". Consider the surface of these conformal squares glued along the corresponding edges. This surface is locally flat but can have cone-like singularities at vertices. If the map is an immersion then the corresponding surface does not have such singularities. Therefore it is more consistent to define as discrete conformal an immersion map on the vertices of the cell decomposition of $C$ which preserves crossratios of its faces.

\section{Proposition 1. [2, 7] Constraint (2) is compatible with (1)}

Compatibility is understood as solvability of some Cauchy problem. In particular a solution to (1), (2) in the subset $\mathbf{Z}_{+}^{\mathbf{2}}$ is uniquely determined by its values $f_{1,0}, f_{0,1}$. Indeed, constraint (2) gives $f_{0,0}=0$ and defines $z$ along the coordinate axis $(n, 0),(0, m)$ as a second-order difference equation. Then all other $f_{k, m}$ with $(k, m) \in \mathbf{Z}_{+}^{\mathbf{2}}$ are calculated through cross-ratios (1).

In this paper the more general initial conditions are considered:

$$
f_{1,0}=1, f_{0,1}=e^{i \beta}
$$

with real $\beta$. For $\alpha=\pi / 2$ this map was studied in detail in [1, 2]. It defines a particular circle pattern with square grid combinatorics introduced by Schramm in [19]. For any $0<\alpha<\pi$ the map is a generalization of Schramm circle patterns.

In what follows we say that the triangle $\left(z_{1}, z_{2}, z_{3}\right)$ has positive (negative) orientation if

$$
\frac{z_{3}-z_{1}}{z_{2}-z_{1}}=\left|\frac{z_{3}-z_{1}}{z_{2}-z_{1}}\right| e^{i \phi} \text { with } 0 \leq \phi \leq \pi \quad(-\pi<\phi<0) .
$$

LEMMA 1. Let $q\left(z_{1}, z_{2}, z_{3}, z_{4}\right)=e^{-2 i \alpha}, 0<\alpha<\pi$.

- If $\left|z_{1}-z_{2}\right|=\left|z_{1}-z_{4}\right|$ and the triangle $\left(z_{1}, z_{2}, z_{4}\right)$ has positive orientation then $\left|z_{3}-z_{2}\right|=\left|z_{3}-z_{4}\right|$ and the angle between $\left[z_{1}, z_{2}\right]$ and $\left[z_{2}, z_{3}\right]$ is $(\pi-\alpha)$.

- If $\left|z_{1}-z_{2}\right|=\left|z_{1}-z_{4}\right|$ and the triangle $\left(z_{1}, z_{2}, z_{4}\right)$ has negative orientation then $\left|z_{3}-z_{2}\right|=\left|z_{3}-z_{4}\right|$ and the angle between $\left[z_{1}, z_{2}\right]$ and $\left[z_{2}, z_{3}\right]$ is $\alpha$.

- If the angle between $\left[z_{1}, z_{2}\right]$ and $\left[z_{1}, z_{4}\right]$ is $\alpha$ and the triangle $\left(z_{1}, z_{2}, z_{4}\right)$ has positive orientation then $\left|z_{3}-z_{2}\right|=\left|z_{1}-z_{2}\right|$ and $\left|z_{3}-z_{4}\right|=\left|z_{4}-z_{1}\right|$.

- If the angle between $\left[z_{1}, z_{2}\right]$ and $\left[z_{1}, z_{4}\right]$ is $(\pi-\alpha)$ and the triangle $\left(z_{1}, z_{2}, z_{4}\right)$ has negative orientation then $\left|z_{3}-z_{2}\right|=\left|z_{1}-z_{2}\right|$ and $\left|z_{3}-z_{4}\right|=\left|z_{4}-z_{1}\right|$.

Proof. straightforward. 
Proposition 2. All the elementary quadrilaterals $\left(f_{n, m}, f_{n+1, m}, f_{n+1, m+1}, f_{n, m+1}\right)$ for the solution of (1), (2) with initial (4) are of kite form: all edges at the vertex $f_{n, m}$ with $n+m=0(\bmod 2)$ are of the same length. Moreover, each elementary quadrilateral has one of the forms enumerated in Lemma 1.

Proof. Given initial $f_{0,1}$ and $f_{1,0}$ constraint (2) allows one to compute $f_{n, 0}$ and $f_{0, m}$ for all $n, m \geq 1$. Induction gives the following equidistant property:

$$
f_{2 n, 0}-f_{2 n-1,0}=f_{2 n+1,0}-f_{2 n, 0}, \quad f_{0,2 m}-f_{0,2 m-1}=f_{0,2 m+1}-f_{0,2 m}
$$

for any $n \geq 1, m \geq 1$. Now using (1) one can successively compute $f_{n, m}$ for any $n, m \in \mathbf{N}$. Lemma 1 completes the proof by induction .

Proposition 2 implies that for $n+m=0(\bmod 2)$ the points $f_{n \pm 1, m} f_{n, m \pm 1}$ lie on the circle with center at $f_{n, m}$. For most $\beta$ (namely for $\beta \neq \alpha$ ) the behavior of the circle pattern thus obtained is quite irregular: interiors of different elementary quadrilaterals intersect.

Definition 3. A discrete map $f_{n, m}$ is called an immersion if interiors of adjacent elementary quadrilaterals $\left(f_{n, m}, f_{n+1, m}, f_{n+1, m+1}, f_{n, m+1}\right)$ are disjoint.

Consider the sublattice $\{n, m: n+m=0(\bmod 2)\}$ and denote by $\mathbf{V}$ its quadrant

$$
\mathbf{V}=\left\{z=N+i M: N, M \in \mathbf{Z}^{2}, M \geq|N|\right\},
$$

where

$$
N=(n-m) / 2, \quad M=(n+m) / 2 .
$$

We use complex labels $z=N+i M$ for this sublattice. Denote by $C(z)$ the circle of radius

$$
R_{z}=\left|f_{n, m}-f_{n+1, m}\right|=\left|f_{n, m}-f_{n, m+1}\right|=\left|f_{n, m}-f_{n-1, m}\right|=\left|f_{n, m}-f_{n, m-1}\right|
$$

with center at $f_{N+M, M-N}=f_{n, m}$.

Let $\{C(z)\}, z \in \mathbf{V}$ be a square grid circle pattern on the complex plane. Define $f_{n, m}: \mathbf{Z}_{+}^{\mathbf{2}} \rightarrow \mathbf{C}$ as follows:

a) if $n+m=0(\bmod 2)$ then $f_{n, m}$ is the center of $C\left(\frac{n-m}{2}+i \frac{n+m}{2}\right)$,

b) if $n+m=1 \quad(\bmod 2)$ then $f_{n, m}:=C\left(\frac{n-m-1}{2}+i \frac{n+m-1}{2}\right) \cap C\left(\frac{n-m+1}{2}+\right.$ $\left.i \frac{n+m+1}{2}\right)=C\left(\frac{n-m+1}{2}+i \frac{n+m-1}{2}\right) \cap C\left(\frac{n-m-1}{2}+i \frac{n+m+1}{2}\right)$. Since all elementary quadrilaterals $\left(f_{n, m}, f_{n+1, m}^{2}, f_{n+1, m+1}, f_{n, m+1}\right)$ are of kite form equation (1) is satisfied automatically. In what follows the function $f_{n, m}$, defined as above by a) and b) is called a discrete map corresponding to the circle pattern $\{C(z)\}$.

Proposition 3. Let the solution of (1), (2)with initial conditions (4) be an immersion, then $R(z)$ defined by (6) satisfies the following equations:

$$
\begin{aligned}
& -M R_{z} R_{z+1}+(N+1) R_{z+1} R_{z+1+i}+(M+1) R_{z+1+i} R_{z+i}-N R_{z+i} R_{z} \\
& \quad=\frac{\gamma}{2}\left(R_{z}+R_{z+1+i}\right)\left(R_{z+1}+R_{z+i}\right)
\end{aligned}
$$

for $z \in \mathbf{V}_{l}:=\mathbf{V} \cup\{-N+i(N-1) \mid N \in \mathbf{N}\}$ and 

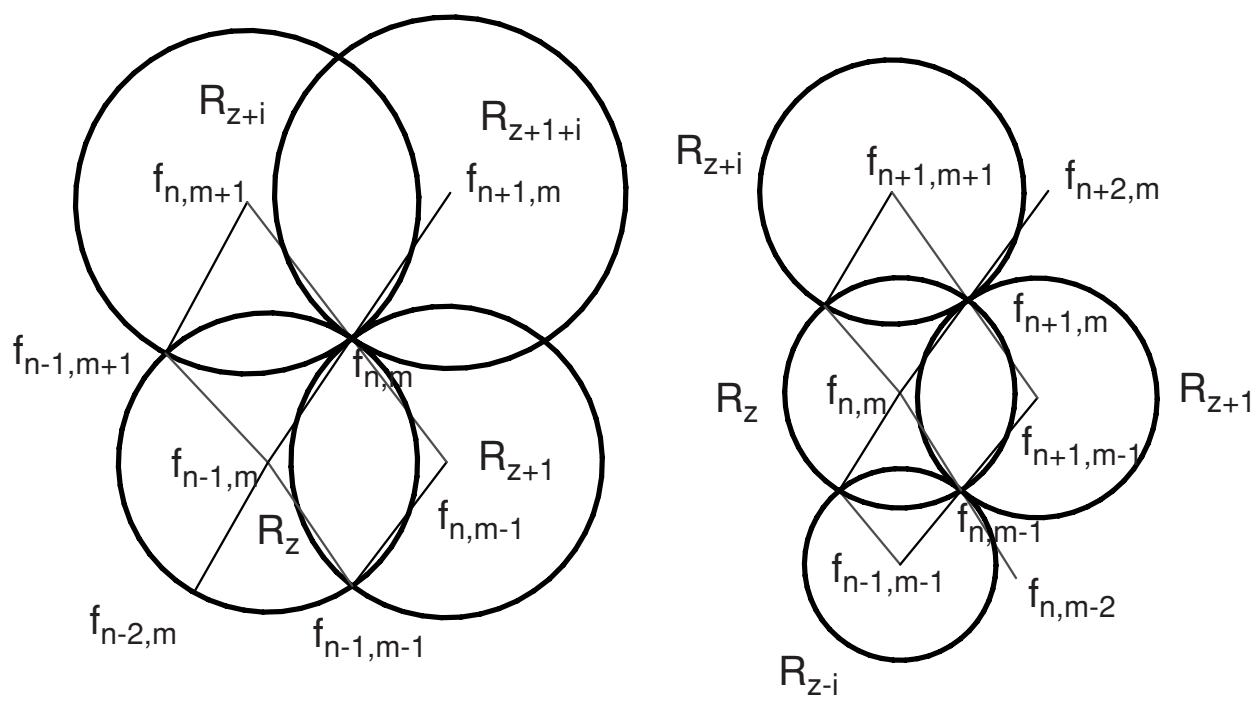

Figure 3. Kite-quadrilaterals of circle pattern

$$
\begin{aligned}
& (N+M)\left(R_{z+i}+R_{z+1}\right)\left(R_{z}^{2}-R_{z+1} R_{z-i}+\cos \alpha R_{z}\left(R_{z-i}-R_{z+1}\right)\right) \\
& \quad+(M-N)\left(R_{z-i}+R_{z+1}\right)\left(R_{z}^{2}-R_{z+1} R_{z+i}+\cos \alpha R_{z}\left(R_{z+i}-R_{z+1}\right)\right)=0,
\end{aligned}
$$

for $z \in \mathbf{V}_{\text {rint }}:=\mathbf{V} \backslash\{ \pm N+i N \mid N \in \mathbf{N}\}$.

Conversely let $R(z): \mathbf{V} \rightarrow \mathbf{R}_{+}$satisfy (7) for $z \in \mathbf{V}_{l}$ and (8) for $z \in \mathbf{V}_{\text {rint }}$. Then $R(z)$ define a square grid circle patterns with intersection angles $\alpha$, the corresponding discrete map $f_{n, m}$ is an immersion and satisfies (1), (2).

Proof. A circle pattern is immersed if and only if all triangles $\left(f_{n, m}, f_{n+1, m}, f_{n, m+1}\right)$ of elementary quadrilaterals of the $\operatorname{map} f_{n, m}$ have the same orientation (for brevity we call it orientation of quadrilaterals). Suppose that the quadrilateral $\left(f_{0,0}, f_{1,0}, f_{1,1}, f_{0,1}\right)$ has positive orientation. Let the circle pattern $f_{n, m}$ be an immersion. For $n+m \equiv$ $1(\bmod 2)$ points $f_{n, m}, f_{n-1, m+1}, f_{n-2, m}, f_{n-1, m-1}$ lie on a circle with center at $f_{n-1, m}$ and radius $R_{z}$, where $z=(n-m-1) / 2+i(n+m-1) / 2$ (See the left part of Fig. 3). Using equation (1) one can compute $f_{n, m+1}$ and $f_{n, m-1}$. Lemma 1 and proposition 2 imply that $f_{n+1, m}$ is in line with $f_{n-1, m}, f_{n, m}$ and that the points $f_{n, m+1}, f_{n, m}, f_{n, m-1}$ are collinear. Denote by $R_{z+1}, R_{z+i}$ the radii of the circle at $f_{n, m-1}$ and $f_{n, m+1}$ respectively and $R_{z+1+i}=R_{z} \frac{\left(f_{n+1, m}-f_{n, m}\right)}{\left(f_{n, m}-f_{n-1, m}\right)}$. Let (2) be satisfied at $(n-1, m)$. Then $(2)$ at $(n, m)$ is equivalent to (7), $R_{z+1+i}$ being positive iff the quadrilaterals $\left(f_{n, m}, f_{n+1, m}, f_{n+1, m+1}, f_{n, m+1}\right)$ and $\left(f_{n, m-1}, f_{n+1, m-1}, f_{n+1, m}, f_{n, m}\right)$ have positive orientation.

Similarly starting with $(2)$ at $(n, m-1)$, where $n+m \equiv 0(\bmod 2)$ (see the right part of Fig. 3) one can determine evolution of the cross-like figure formed by $f_{n, m-1}, f_{n+1, m-1}, f_{n, m}, f_{n-1, m-1}, f_{n, m-2}$ into $f_{n+1, m}, f_{n+2, m}, f_{n+1, m+1}, f_{n, m}, f_{n+1, m-1}$. Equation (2) at $(n+1, m)$ is equivalent to (7) and (8) at $z=(n-m) / 2+i(n+m) / 2$. $R_{z+1}$ is positive only for an immersed circle pattern.

Now let $R_{z}$ be some positive solution to (7), (8). We can rescale it so that $R_{0}=1$. This solution is completely defined by $R_{0}, R_{i}$. Consider solution $f_{n, m}$ of (1), (2) with 
initial data (4) where $\beta$ is chosen so that the quadrilateral $\left(f_{0,0}, f_{1,0}, f_{1,1}, f_{0,1}\right)$ has positive orientation and satisfies the conditions $R_{0}=1=\left|f_{0,0}-f_{1,0}\right|$ and $R_{i}=\left|f_{1,1}-f_{1,0}\right|$. The $\operatorname{map} f_{n, m}$ defines circle pattern due to proposition 2 . It can be uniquely computed from these equations. To this end one has to resolve (7) with respect to $R_{z+i+1}$ and use it to find $f_{n+1, m}$ from $R_{z+1+i}=\frac{R_{z}\left(f_{n+1, m}-f_{n, m}\right)}{\left(f_{n, m}-f_{n-1, m}\right)}$ and to resolve (8) for $R_{z+i}$ to find $f_{n+1, m+1}$ from $R_{z+i}=R_{z+1} \frac{\left(f_{n+1, m+1}-f_{n+1, m}\right)}{\left(f_{n+1, m}-f_{n+1, m-1}\right)}$. One can reverse the argument used in the derivation of (7), (8) to show that $f$ satisfies (1), (2). Moreover, since $R_{z}$ is positive, at each step we get positively orientated quadrilaterals.

Note that initial data (4) for $f_{n, m}$ imply initial data for $R_{z}$ :

$$
R_{0}=1, \quad R_{i}=\frac{\sin \frac{\beta}{2}}{\sin \left(\alpha-\frac{\beta}{2}\right)} .
$$

Definition 4. A discrete map $f_{n, m}$ is called embedded if interiors of different elementary quadrilaterals $\left(f_{n, m}, f_{n+1, m}, f_{n+1, m+1}, f_{n, m+1}\right)$ do not intersect.

THEOREM 1. If for a solution $R_{z}$ of (7), (8) with $\gamma \neq 1$ and initial conditions (9) it holds that

$$
R_{z}>0, \quad(\gamma-1)\left(R_{z}^{2}-R_{z+1} R_{z-i}+\cos \alpha R_{z}\left(R_{z-i}-R_{z+1}\right)\right) \geq 0
$$

in $\mathbf{V}_{\text {int }}$, then the corresponding discrete map is embedded.

The proof of this theorem for the case $\alpha=\pi / 2$ is given in [1]). For generic $\alpha$ it is the same with obvious modifications.

3. Discrete Riccati equation and hypergeometric functions. Let $r_{n}$ and $R_{n}$ be radii of circles with centers at $f_{2 n, 0}, f_{2 n+1,1}$ respectively (see Fig. 4).

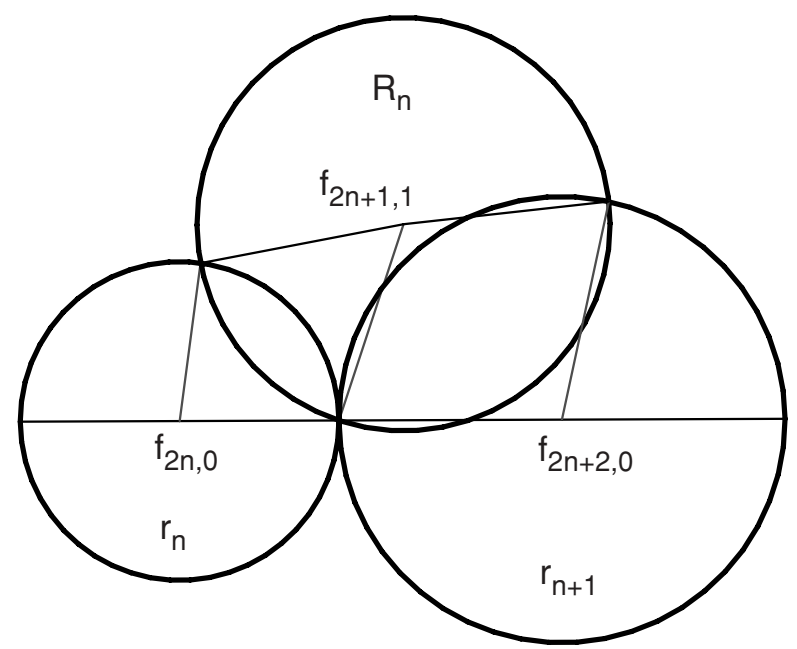

Figure 4. Circles on the border 
Constraint (2) and property (5) give

$$
r_{n+1}=\frac{2 n+\gamma}{2(n+1)-\gamma} r_{n} .
$$

From elementary geometric considerations one gets

$$
R_{n+1}=\frac{r_{n+1}-R_{n} \cos \alpha}{R_{n}-r_{n+1} \cos \alpha} r_{n+1}
$$

Define

$$
p_{n}=\frac{R_{n}}{r_{n}}, \quad g_{n}(\gamma)=\frac{2 n+\gamma}{2(n+1)-\gamma}
$$

and denote $t=\cos \alpha$ for brevity. Now the equation for radii $R, r$ takes the form:

$$
p_{n+1}=\frac{g_{n}(\gamma)-t p_{n}}{p_{n}-\operatorname{tg}_{n}(\gamma)} .
$$

REMARK. Equation (11) is a discrete version of the Riccati equation. This nomenclature is motivated by the following properties:

- the cross-ratio of each four-tuple of its solutions is constant since $p_{n+1}$ is a Möbius transform of $p_{n}$,

- the general solution is expressed in terms of the solution of some linear equation (see this linearisation below).

Below we call (11) the d-Riccati equation.

THEOREM 2. The solution of the discrete Riccati equation (11) is positive for $n \geq 0$ if and only if

$$
p_{0}=\frac{\sin \frac{\gamma \alpha}{2}}{\sin \frac{(2-\gamma) \alpha}{2}} .
$$

The proof is based on the closed form of the general solution of the d-Riccati linearisation. It is linearised by the standard Ansatz

$$
p_{n}=\frac{y_{n+1}}{y_{n}}+\operatorname{tg}_{n}(\gamma)
$$

which transforms it into

$$
y_{n+2}+t\left(g_{n+1}(\gamma)+1\right) y_{n+1}+\left(t^{2}-1\right) g_{n}(\gamma) y_{n}=0 \text {. }
$$

One can guess that there is only one initial value $p_{0}$ giving a positive d-Riccati solution from the following consideration: $g_{n}(\gamma) \rightarrow 1$ as $n \rightarrow \infty$, and the general solution of equation (14) with limit values of the coefficients is $y_{n}=c_{1}(-1)^{n}(1+t)^{n}+c_{2}(1-t)^{n}$. So $p_{n}=\frac{y_{n+1}}{y_{n}}+\operatorname{tg}_{n}(\gamma) \rightarrow-1$ for $c_{1} \neq 0$. However $c_{1}, c_{2}$ define only asymptotics of a solution. To relate it to initial values one needs some kind of connection formulas. Fortunately it is possible to find the general solution to (14). 
Proposition 4. The general solution to (14) is

$$
\begin{aligned}
y_{n}= & \frac{\Gamma\left(n+\frac{1}{2}\right)}{\Gamma\left(n+1-\frac{\gamma}{2}\right)}\left(c_{1} \lambda_{1}^{n+1-\gamma / 2} F\left(\frac{3-\gamma}{2}, \frac{\gamma-1}{2}, \frac{1}{2}-n, z_{1}\right)\right. \\
& \left.+c_{2} \lambda_{2}^{n+1-\gamma / 2} F\left(\frac{3-\gamma}{2}, \frac{\gamma-1}{2}, \frac{1}{2}-n, z_{2}\right)\right)
\end{aligned}
$$

where $\lambda_{1}=-t-1, \lambda_{2}=1-t, z_{1}=(t-1) / 2, z_{2}=-(1+t) / 2$ and $F$ stands for the hypergeometric function.

Proof. Solutions are found by a slightly modified symbolic method (see [9] for the method's description). The substitution

$$
y_{n}=u_{x} \lambda^{x}, \quad x=n+1-\gamma / 2
$$

transforms (14) into

$$
\lambda^{2}(x+1) x u_{x+2}+2 t\left(x+\frac{\gamma+1}{2}\right) x u_{x+1}+\left(t^{2}-1\right)(x+\gamma-1)(x+1) u_{x}=0 .
$$

We are looking for a solution in the form

$$
u_{x}=\sum_{m=-\infty}^{\infty} a_{m} v_{x, m}
$$

where $v_{x, m}$ satisfies

$$
(x+m) v_{x, m}=v_{x, m+1}, \quad x v_{x+1, m}=v_{x, m+1} .
$$

REMARK. Note that the label $m$ in (18) augments by 1 but is not necessarily an integer and therefore $v_{x, m}$ is a straightforward generalization of $x^{(m)}=$ $(x+m-1)(x+m-2) \ldots(x+1) x$ playing the role of $x^{m}$ in the calculus of finite differences. The general solution to (19) is expressed in terms of the $\Gamma$-function:

$$
v_{x, m}=c \frac{\Gamma(x+m)}{\Gamma(x)}
$$

The Stirling formula for large $x$

$$
\Gamma(x) \simeq \sqrt{2 \pi} e^{-x} x^{x-\frac{1}{2}}
$$

gives the asymptotics for $v_{x, m}$ :

$$
v_{x, m} \simeq c x^{m} \text { for } x \rightarrow \infty .
$$

Substituting (18) into (17), making use of (19) and collecting similar terms one gets the following equation for the coefficients:

$$
\begin{aligned}
& \left(\lambda^{2}+2 t \lambda+t^{2}-1\right) a_{m-2}+2\left(\frac{1+\gamma}{2}-m\right)\left(t \lambda+t^{2}-1\right) a_{m-1} \\
& \quad+\left(t^{2}-1\right)(1-m)(\gamma-1-m) a_{m}=0
\end{aligned}
$$


Choosing $\lambda_{1}=-t-1$ or $\lambda_{2}=1-t$ kills the term containing $a_{m-2}$. To make the series (18) convergent we can use the freedom in $m$ to truncate (18) on one side. The choice $m \in \mathbf{Z}$ or $m \in \gamma+\mathbf{Z}$ leads to divergent series. For $m \in \frac{\gamma+1}{2}+\mathbf{Z}$ equation (23) gives $a_{\frac{\gamma+1}{2}+k}=0$ for all non-negative integer $k$ and

$$
a_{\frac{\gamma+1}{2}-k-1}=\frac{1-t^{2}}{t \lambda+t^{2}-1} \frac{\left(k-\frac{\gamma-1}{2}\right)\left(k-1+\frac{\gamma-1}{2}\right)}{2 k} a_{\frac{\gamma+1}{2}-k}
$$

where $\lambda=\lambda_{1}, \lambda_{2}$. Substitution of the solution of this recurrent relation in terms of the $\Gamma$-functions and (20) yields

$$
y_{x}=\lambda^{x} \sum_{k=1}^{\infty}\left(\frac{1-t^{2}}{2\left(t \lambda+t^{2}-1\right)}\right)^{k} \frac{\Gamma\left(k-\frac{\gamma-1}{2}\right) \Gamma\left(k-1+\frac{\gamma-1}{2}\right) \Gamma\left(x+\frac{\gamma+1}{2}-k\right)}{\Gamma(k) \Gamma(x)}
$$

LEMMA 2. For both $\lambda=-t-1,1-t$ the series (25) converges for all $x$.

Proof of Lemma 2. Since $z=\frac{1-t^{2}}{2\left(t \lambda+t^{2}-1\right)}=(t-1) / 2,-(1+t) / 2$ for $\lambda_{1}, \lambda_{2}$ respectively and $t=\cos \alpha<1$ the convergence of (25) depends on the behavior of $\frac{\Gamma\left(k-\frac{\gamma-1}{2}\right) \Gamma\left(k-1+\frac{\gamma-1}{2}\right) \Gamma\left(x+\frac{\gamma+1}{2}-k\right)}{\Gamma(k)}$. Stirling's formula (21) ensures that this expression is bounded by $c k^{\phi(x, \gamma)}$ for some $c$ and $\phi(x, \gamma)$ which gives convergence.

Series (25) is expressed in terms of hypergeometric functions:

$$
y_{x}=\lambda^{x} \frac{\Gamma\left(x+\frac{\gamma-1}{2}\right) \Gamma\left(1-\frac{\gamma-1}{2}\right) \Gamma\left(\frac{\gamma-1}{2}\right)}{\Gamma(x)} F\left(1-\frac{\gamma-1}{2}, \frac{\gamma-1}{2}, 1-\left(x+\frac{\gamma-1}{2}\right), z\right)
$$

where

$$
\begin{aligned}
& F\left(1-\frac{\gamma-1}{2}, \frac{\gamma-1}{2}, 1-\left(x+\frac{\gamma-1}{2}\right), z\right)=1+z \frac{\left(1-\frac{\gamma-1}{2}\right)\left(\frac{\gamma-1}{2}\right)}{\left(1-\left(x+\frac{\gamma-1}{2}\right)\right)}+\cdots \\
& +z^{k} \frac{\left[\left(1-\frac{\gamma-1}{2}\right)\left(2-\frac{\gamma-1}{2}\right) \cdots\left(k-\frac{\gamma-1}{2}\right)\right]\left[\left(\frac{\gamma-1}{2}\right)\left(1+\frac{\gamma-1}{2}\right) \cdots\left(k-1+\frac{\gamma-1}{2}\right)\right]}{\left(1-\left(x+\frac{\gamma-1}{2}\right)\right) \cdots\left(k-\left(x+\frac{\gamma-1}{2}\right)\right)}+\cdots
\end{aligned}
$$

Here the standard designation $F(a, b, c, z)$ for the hypergeometric function as a solution holomorphic at $z=0$ for the equation

$$
z(1-z) F_{z z}+[c-(a+b+1) z] F_{z}-a b F=0
$$

is used.

Now we can complete the proof of Proposition 4. Due to linearity the general solution of (14) is given by superposition of any two linearly independent solutions. As was shown each summond in (15) satisfies the equation (14). To finish the proof of Proposition 4 one has to show that the particular solutions with $c_{1}=0, c_{2} \neq 0$ and $c_{1} \neq 0, c_{2}=0$ are linearly independent, which follows from the following Lemma.

LeMMA 3. As $n \rightarrow \infty$ function (15) has the asymtotics

$$
y_{n} \simeq(n+1-\gamma / 2)^{\frac{\gamma-1}{2}}\left(c_{1} \lambda_{1}^{n+1-\gamma / 2}+c_{2} \lambda_{2}^{n+1-\gamma / 2}\right)
$$


Proof. For $n \rightarrow \infty$ the series representation (26) gives $F\left(\frac{3-\gamma}{2}, \frac{\gamma-1}{2}, \frac{1}{2}-n, z_{1}\right) \simeq 1$. Stirling's formula (21) defines the asymptotics of the factor $\frac{\Gamma\left(n+\frac{1}{2}\right)}{\Gamma\left(n+1-\frac{\gamma}{2}\right)}$ (compare with (22)).

Proof of Theorem 2: For positive $p_{n}$ it is necessary that $c_{1}=0$. This follows from asymptotics (28) substituted into (13). Let us define

$$
\begin{aligned}
s(z)= & 1+z \frac{\left(1-\frac{\gamma-1}{2}\right)\left(\frac{\gamma-1}{2}\right)}{\frac{1}{2}}+\cdots \\
& +z^{k} \frac{\left(k-\frac{\gamma-1}{2}\right) \cdots\left(1-\frac{\gamma-1}{2}\right)\left(\frac{\gamma-1}{2}\right)\left(k-1+\frac{\gamma-1}{2}\right)}{k !\left(k-\frac{1}{2}\right) \cdots \frac{1}{2}} \cdots
\end{aligned}
$$

This is the hypergeometric function $F\left(\frac{3-\gamma}{2}, \frac{\gamma-1}{2}, \frac{1}{2}-n, z\right)$ with $n=0$. A straightforward manipulation with series shows that

$$
p_{0}=1+\frac{2(\gamma-1)}{2-\gamma} z+\frac{4 z(z-1)}{2-\gamma} \frac{s^{\prime}(z)}{s(z)}
$$

where $z=\frac{1+t}{2}$. Note that $p_{0}$ as a function of $z$ satisfies an ordinary differential equation of first order since $\frac{s^{\prime}(z)}{s(z)}$ satisfies Riccati equation obtained by reduction of (27). Computation shows that $\frac{\sin \frac{\gamma \alpha}{2}}{\sin \frac{(2-\gamma) \alpha}{2}}$ satisfies the same ODE. Since both expression (30) and (12) are equal to 1 for $z=0$ they coincide everywhere.

COROLlary 1. If there exists immersed $f_{n, m}$ satisfying (1), (2), (4) it is defined by initial data (3).

4. Embedded circle patterns and discrete Painlevé equations. Let $R_{z}$ be a solution of (7) and (8) with initial condition (9). For $z \in \mathbf{V}_{\text {int }}$ define $P_{N, M}=P_{z}=\frac{R_{z+1}}{R_{z-i}}, Q_{N, M}=$ $Q_{z}=\frac{R_{z}}{R_{z-i}}$. Then (8) and (7) are rewritten as follows

$$
\begin{aligned}
Q_{N, M+1} & =\frac{(N-M) Q_{N, M}\left(1+P_{N, M}\right)\left(Q_{N, M}-P_{N, M} \cos \alpha\right)-(M+N) P_{N, M} S_{N, M}}{Q_{N, M}\left[(M+N) S_{N, M}-(M-N)\left(1+P_{N, M}\right)\left(P_{N, M}-Q_{N, M} \cos \alpha\right)\right]}, \\
P_{N, M+1} & =\frac{(2 M+\gamma) P_{N, M}+(2 N+\gamma) Q_{N, M} Q_{N, M+1}}{(2(N+1)-\gamma) P_{N, M}+(2(M+1)-\gamma) Q_{N, M} Q_{N, M+1}},
\end{aligned}
$$

where

$$
S_{N, M}=Q_{N, M}^{2}-P_{N, M}+Q_{N, M}\left(1-P_{N, M}\right) \cos \alpha .
$$

Property (10) for (31), (32) reads as

$$
(\gamma-1)\left(Q_{N, M}^{2}-P_{N, M}+Q_{N, M}\left(1-P_{N, M}\right) \cos \alpha\right) \geq 0, Q_{N, M}>0, P_{N, M}>0 .
$$

Equations (31), (32) can be considered as a dynamical system in the variable $M$.

THEOREM 3. There exists $a>0$ such that (10) holds for the solution $R_{z}$ of (7), (8) with initial conditions

$$
R_{0}=1, \quad R_{i}=a
$$




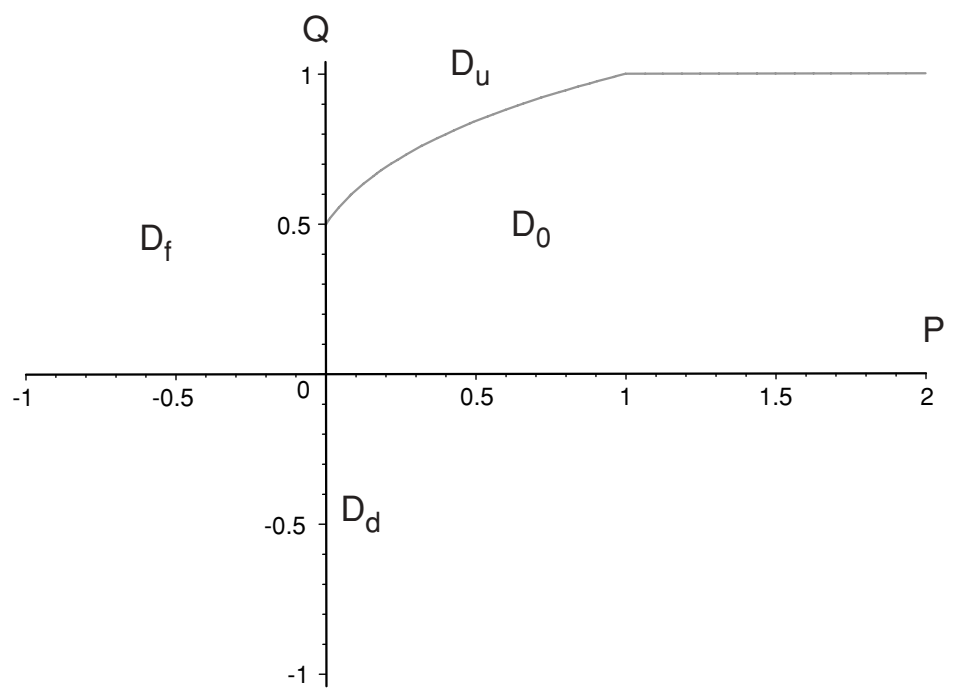

Figure 5. The case $\cos \alpha=-1 / 2$

Proof. Almost literally that for the case $\alpha=\pi / 2$ [1]. Due to the following Lemma it is sufficient to prove (10) only for $0<\gamma<1$.

LEMMA 4. If $R_{z}$ is a solution of (7), (8) for $\gamma$ then $1 / R_{z}$ is a solution of $(7,8)$ for $\tilde{\gamma}=2-\gamma$.

The lemma is proved by straightforward computation.

Let $0<\gamma<1$ and let $\left(P_{N, M}, Q_{N, M}\right)$ correspond to the solution of (7), (8) with initial conditions (34). Define a real function $F(P)$ on $\mathbf{R}_{+}$implicitly by $F^{2}-P+$ $F(1-P) \cos \alpha=0$ for $0 \leq P \leq 1$ and by $F(P) \equiv 1$ for $1 \leq P$.

Designate

$$
\begin{aligned}
& D_{u}:=\{(P, Q): P>0, Q>F(P)\}, \quad D_{d}:=\{(P, Q): Q<0\}, \\
& D_{0}:=\{(P, Q): P>0,0 \leq Q \leq F(P)\}, \quad D_{f}:=\{(P, Q): P \leq 0, Q \geq 0\}
\end{aligned}
$$

as in Fig. 5. Now define the infinite sequences $\left\{q_{n}\right\},\left\{p_{n}\right\}, n \in \mathbf{N}$ as follows:

$$
\begin{aligned}
& \left\{q_{n}(a)\right\}:=\left\{Q_{0,1}, Q_{0,2}, Q_{1,2}, Q_{0,3}, Q_{1,3}, Q_{2,3}, \ldots, Q_{0, M}, Q_{1, M}, \ldots, Q_{M-1, M}, \ldots\right\}, \\
& \left\{p_{n}(a)\right\}:=\left\{P_{0,1}, P_{0,2}, P_{1,2}, P_{0,3}, P_{1,3}, P_{2,3}, \ldots, P_{0, M}, P_{1, M}, \ldots, P_{M-1, M}, \ldots\right\} .
\end{aligned}
$$

and the sets

$$
\begin{aligned}
& A_{u}(n):=\left\{a \in \mathbf{R}_{+}:\left(p_{n}(a), q_{n}(a)\right) \in D_{u},\left(p_{k}(a), q_{k}(a)\right) \in D_{0} \forall 0<k<n\right\}, \\
& A_{d}(n):=\left\{a \in \mathbf{R}_{+}:\left(p_{n}(a), q_{n}(a)\right) \in D_{d},\left(p_{k}(a), q_{k}(a)\right) \in D_{0} \forall 0<k<n\right\} .
\end{aligned}
$$

$A_{u}(n)$ and $A_{d}(n)$ are open sets since the denominators of (31), (32) do not vanish in $D_{0}$. Moreover, direct computation shows that $A_{u}(1) \neq \emptyset$ and $A_{d}(2) \neq \emptyset$, therefore the sets

$$
A_{u}:=\cup A_{u}(k), \quad A_{d}:=\cup A_{d}(k)
$$


are not empty. Finally, define

$$
A_{0}:=\left\{a \in \mathbf{R}_{+}:\left(p_{n}(a), q_{n}(a)\right) \in D_{0}, \forall n \in \mathbf{N}\right\} .
$$

Note that $A_{0}, A_{u}, A_{d}$ are mutually disjoint and the sequences $\left\{p_{n}\right\},\left\{q_{n}\right\}$ is so constructed that

$$
\mathbf{R}_{+}=A_{0} \cup A_{u} \cup A_{d} .
$$

Indeed $\left(P_{N, M}, Q_{N, M}\right)$ cannot jump from $D_{0}$ into $D_{f}$ in one step $M \rightarrow M+1$ since $P_{N, M+1}$ is positive for positive $P_{N, M}, Q_{N, M}, Q_{N, M+1}$. Relation (35) would be impossible for $A_{0}=\emptyset$, since the connected set $\mathbf{R}_{+}$cannot be covered by two open disjoint nonempty subsets $A_{u}$ and $A_{d}$. Therefore $A_{0} \neq \emptyset$.

THEOREM 4. The discrete map $Z^{\gamma}, 0<\gamma<2$, is embedded.

Proof. Theorems 3 and 1 ensure that there is an embedded $Z^{\gamma}$ for each $a \in A_{0}$. Theorem 2 implies that the set $A_{0}$ consists of only one element, namely, $A_{0}=\left\{\sin \frac{\gamma \alpha}{2} / \sin \frac{(2-\gamma) \alpha}{2}\right\}$.

Remark 1. For $N=0$ the system (31), (32) for $Q_{N, M}, P_{N, M}$ reduces to the special case of the discrete Painlevé equation (compare with [1]):

$$
(n+1)\left(x_{n}^{2}-1\right)\left(\frac{x_{n+1}+x_{n} / \varepsilon}{\varepsilon+x_{n} x_{n+1}}\right)-n\left(1-x_{n}^{2} / \varepsilon^{2}\right)\left(\frac{x_{n-1}+\varepsilon x_{n}}{\varepsilon+x_{n-1} x_{n}}\right)=\gamma x_{n} \frac{\varepsilon^{2}-1}{2 \varepsilon^{2}},
$$

where $\varepsilon=e^{i \alpha}$. This equation allows us to represent $x_{n+1}$ as a function of $n, x_{n-1}$ and $x_{n}: x_{n+1}=\Phi\left(n, x_{n-1}, x_{n}\right) . \Phi(n, u, v)$ maps the torus $T^{2}=S^{1} \times S^{1}=\{(u, v) \in \mathbf{C}:|u|=$ $|v|=1\}$ into $S^{1}$ and has the following properties:

- $\forall n \in \mathbf{N}$ it is a continuous map on $A_{I} \times A_{I}$ where $A_{I}=\left\{e^{i \beta}: \beta \in[0, \alpha]\right\}$.

- For $(u, v) \in A_{I} \times A_{I} \Phi(n, u, v) \in A_{I} \cup A_{I I} \cup A_{I V}$ holds true, where $A_{I I}=\left\{e^{i \beta}\right.$ : $\beta \in(\alpha, \pi]\}$ and $A_{I V}=\left\{e^{i \beta}: \beta \in[\alpha-\pi, 0)\right\}$, i.e. $x$ cannot jump in one step from $A_{I}$ into $A_{I I I}=\left\{e^{i \beta}: \beta \in(-\pi, \alpha-\pi)\right\}$.

These properties guarantee that there exists a unitary solution $x_{n}=e^{i \alpha_{n}}$ of this equation with $x_{0}=e^{i \gamma \alpha / 2}$ in the sector $0<\alpha_{n}<\alpha$. This solution corresponds to an embedded $Z^{\gamma}$. Equation (36) is a special case of a more general reduction of the cross-ratio equation (see [16], [2] for details).

REMARK 2. Discrete maps $Z^{2}$ and Log are defined for $\alpha \neq \pi / 2$ with the aid of limit renormalization of $Z^{\gamma}$ as $\gamma \rightarrow 2-0$ and as dual to $Z^{2}$ respectively as in [2], where the reader can find details for the case $\alpha=\pi / 2$.

THEOREM 5. The discrete maps $Z^{2}$ and Log are embedded.

The proof for $\alpha=\pi / 2$ is given in [1]. It does not change for the generalization discussed.

5. Concluding remarks. Further generalizations of discrete $Z^{\gamma}$ and Log are possible.

I. One can relax the unitary condition for cross-ratios and consider solutions to

$$
q\left(f_{n, m}, f_{n+1, m}, f_{n+1, m+1}, f_{n, m+1}\right)=\kappa^{2} e^{-2 i \alpha}
$$




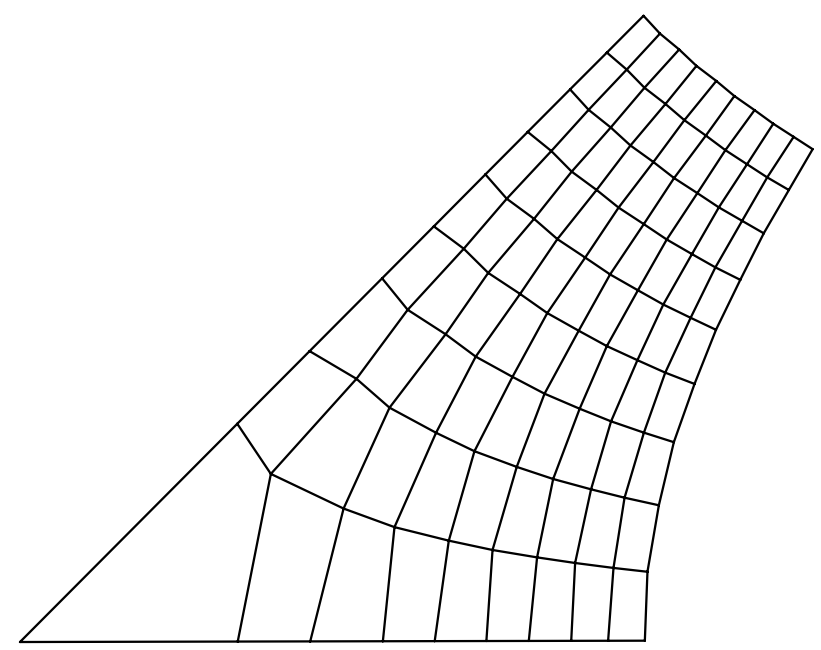

Figure 6. Discrete $Z^{1 / 2}, \kappa=2, \alpha=\pi / 2$

subject to the same constraint (2) with the initial data

$$
f_{1,0}=1, \quad f_{0,1}=\frac{e^{i \gamma \alpha}}{\kappa} .
$$

This solution is a discrete analog of $Z^{\gamma}$ defined on the vertices of regular parallelogram lattice (see Fig. 6). However, the mappings thus obtained are deprived of geometrical flavor as they do not define circle patterns.

II. Another possibility is to deregularize prescribed combinatorics as follows. Consider $\mathbf{Z}_{+}^{\mathbf{n}} \subset \mathbf{R}^{\mathbf{n}}$. For each coordinate vector $\mathbf{e}_{\mathbf{i}}=\left(e_{i}^{1}, \ldots, e_{i}^{n}\right)$ where $e_{i}^{j}=\delta_{i}^{j}$ define a unit vector $\xi_{\mathbf{i}}$ in $\mathbf{C}=\mathbf{R}^{\mathbf{2}}$ so that for any pair of indexes $i, j$ the vectors $\xi_{\mathbf{i}}, \xi_{\mathbf{j}}$ form a basis in $\mathbf{R}^{\mathbf{2}}$. Let $\Omega \in \mathbf{R}^{\mathbf{n}}$ be some 2 -dimensional simply connected cell complex with vertices in $\mathbf{Z}_{+}^{\mathbf{n}}$. Suppose $0 \in \Omega$. (We denote by the same symbol $\Omega$ the set of complex vertices.) Define the map $P: \Omega \rightarrow \mathbf{C}$ by the following conditions:

1) $P(0)=0$,

2) if $x, y$ are vertices of $\Omega$ and $y=x+\mathbf{e}_{\mathbf{i}}$ then $P(y)=P(x)+\xi_{\mathbf{i}}$.

It is easy to see that $P$ is correctly defined and unique.

We call $\Omega$ a projectable cell complex iff its image $\omega=P(\Omega)$ is embedded, i.e. intersections of images of different cells of $\Omega$ do not have interiors.

It is natural to define a discrete conformal map on $\omega$ as a discrete complex immersion function $f$ on vertices of $\omega$ preserving the cross-ratios of $\omega$-cells. The argument of $f$ can be labelled by the vertices $x$ of $\Omega$. Hence for any cell of $\Omega$, the function $f$ constructed on $\mathbf{e}_{\mathbf{k}}, \mathbf{e}_{\mathbf{j}}$ satisfies the following equation for cross-ratios:

$$
q\left(f_{x}, f_{x+\mathbf{e}_{\mathbf{k}}}, f_{x+\mathbf{e}_{\mathbf{k}}+\mathbf{e}_{\mathbf{j}}}, f_{x+\mathbf{e}_{\mathbf{j}}}\right)=e^{-2 i \alpha_{k, j},}
$$

where $\alpha_{k, j}$ is the angle between $\xi_{\mathbf{k}}$ and $\xi_{\mathbf{j}}$, taken positive if $\left(\xi_{\mathbf{k}}, \xi_{\mathbf{j}}\right)$ has positive orientation and taken negative otherwise. Now suppose that $f$ is a solution to (39) defined on the whole of $\mathbf{Z}_{+}^{\mathbf{n}}$. Equation (39) is compatible with the constraint

$$
\gamma f_{x}=\sum_{s=1}^{n} 2 x_{s} \frac{\left(f_{x+\mathbf{e}_{\mathbf{s}}}-f_{x}\right)\left(f_{x}-f_{x-\mathbf{e}_{\mathbf{s}}}\right)}{f_{x+\mathbf{e}_{\mathbf{s}}}-f_{x-\mathbf{e}_{\mathbf{s}}}} .
$$


This constraint could be derived from some discrete isomonodromy problem (for $n=3$ see [7]) which ensures the compatibility. Now we can define a discrete $Z^{\gamma}: \omega \rightarrow \mathbf{C}$ for projectable $\Omega$ as a solution to (39), (40) restricted to $\Omega$. Initial conditions for this solution are of the form (3) so that the restrictions of $f$ on each two-dimensional coordinate lattice is an immersion defining a circle pattern with prescribed intersection angles.

This definition naturally generalizes the definition of discrete $Z^{\gamma}$ given in [7] for $\Omega=\{(k, l, m): k+l+m=0, \pm 1\}$.

\section{Conjecture. Discrete $Z^{\gamma}: \omega \rightarrow \mathbf{C}$ is embedded.}

Schramm [19] showed that there are square grid circle patterns mimicking $\operatorname{Erf}(\sqrt{i} z)$ but that an analogue of $\operatorname{Erf}(z)$ does not exist. The obstacle is purely combinatorial. There is a hope that the combinatorics of projectable cells may give more examples of discrete analogues of classical functions.

ACKNOWLEDGEMENTS. The author thanks R. Halburd for useful discussions. This research was partially supported by the EPSRC grant No Gr/N30941.

\section{REFERENCES}

1. S. I. Agafonov, Embedded circle patterns with the combinatorics of the square grid and discrete Painlevé equations, Discrete Comput. Geom. 29 (2003), 305-319.

2. S. I. Agafonov and A. I. Bobenko, Discrete $Z^{\gamma}$ and Painlevé equations, Internat. Math. Res. Notices 4 (2000), 165-193.

3. A. F. Beardon, T. Dubejko and K. Stephenson, Spiral hexagonal circle packings in the plane, Geom. Dedicata 49 (1994), 39-70.

4. A. F. Beardon and K. Stephenson, The uniformization theorem for circle packings, Indiana Univ. Math. J. 39, No. 4 (1990), 1383-1425.

5. A. I. Bobenko and T. Hoffman, Conformally symmetric circle packings. A generalization of Doyle spirals, Experimental Math. 10, No. 1 (2001), 141-150.

6. A. I. Bobenko, T. Hoffman and Yu. B. Suris, Hexagonal circle patterns and integrable systems. Patterns with the multi-ratio property and Lax equations on the regular triangular lattice, Internat. Math. Res. Notices 3 (2002), 111-164.

7. A. I. Bobenko and T. Hoffman, Hexagonal circle patterns and integrable systems. Patterns with constant angles, Duke Math. J. 116 (2003), 525-566.

8. A. Bobenko and U. Pinkall, Discrete isothermic surfaces, J. Reine Angew. Math. $\mathbf{4 7 5}$ (1996), 187-208.

9. G. Boole, Calculus of finite differences (Chelsea Publishing Company, 1872).

10. K. Callahan and B. Rodin, Circle packing immersions form regularly exhaustible surfaces, Complex Variables 21 (1993), 171-177.

11. T. Dubejko and K. Stephenson, Circle packings: Experiments in discrete analytic function theory, Experimental Math. 4, No. 4 (1995), 307-348.

12. Z. -X. He and O. Schramm, The $C^{\infty}$ convergence of hexagonal disc packings to Riemann map, Acta. Math. 180 (1998), 219-245. 33.

13. Z. -X. He, Rigidity of infinite disk patterns, Annals of Mathematics 149 (1999), 1-

14. T. Hoffman, Discrete CMC surfaces and discrete holomorphic maps, in Discrete Integrable Geometry and Physics (Eds.: A. I. Bobenko and R. Seiler), (Oxford University Press, 1999), 97-112.

15. A. Marden and B. Rodin, On Thurston's formulation and proof of Andreev's theorem, in Computational methods and function theory (Valparaiso, 1989), Lecture Notes in Math. No. 1435 (Springer-Verlag, 1990),103-115. 
16. F. W. Nijhoff, A. Ramani, B. Grammaticos and Y. Ohta, On discrete Painlevé equations associated with lattice KdV systems and the Painlevé VI equation, Stud. Appl. Math. 106 (2001), 261-314.

17. B. Rodin, Schwarz's lemma for circle packings, Invent. Math. 89 (1987), 271-289.

18. B. Rodin and D. Sullivan, The convergence of circle packings to Riemann mapping, J. Diff. Geometry 26 (1987), 349-360.

19. O. Schramm, Circle patterns with the combinatorics of the square grid, Duke Math. J. 86 (1997), 347-389.

20. W. P. Thurston, The finite Riemann mapping theorem, Invited address, International Symposium in Celebration of the Proof of the Bieberbach Conjecture (Purdue University, 1985).

21. A. Zabrodin, A survey of Hirota's difference equation, Teor. Math. Phys. 113 (1997) 1347-1392. 